30. September 2009

\title{
Welche Sprache spricht das Recht? Rechtssprache zwischen Bestimmtheit, Offenheit und geplanter Fehldeutung
}

\author{
Paul KirchHoF
}

\section{Recht lebt in Sprache}

Wenn wir miteinander sprechen, erwarten wir, dass der Partner unsere Worte so versteht, wie wir sie gemeint haben. Wir wählen eine Sprechweise, mit der wir den anderen in der Gemeinschaft einer Sprache erreichen. Auch das Recht wirkt durch Sprache. Wenn das Gesetz eine allgemeinverbindliche Regel setzt, wählt es einen Text, der eine für alle Adressaten gleiche Rechtsfolge begründen soll. Die Gleichheit vor dem Gesetz baut darauf, dass die sprachliche Anordnung von allen Adressaten in gleichem Inhalt verstanden werden kann.

Dieser Sprachoptimismus hat Voraussetzungen: Der Staat findet in der Gemeinsamkeit dank Sprache seine Grundlage für Einheit und Zusammenhalt. Allein die sprachliche Verständigung befähigt eine Gruppe von Menschen, zu einem Staatsvolk zusammenzufinden und damit eine Demokratie zu begründen. Zugleich ist die Sprache Grundlage der individuellen Freiheit: Der Mensch könnte seine Meinung nicht äußern, sich aus den Medien nicht unterrichten, die Kulturfreiheiten von Ehe und Familie, von Kunst und Wissenschaft nicht ausüben, seine politischen Rechte nicht wahrnehmen, wenn er nicht in einer gemeinsamen Sprache hören, sprechen und lesen könnte. Recht begreift die Fragen des Rechts in gemeinsamer Sprache und hält die Antworten in dieser Sprache fest.

Das Recht zum Gebrauch der Muttersprache ist heute ein Menschenrecht, wird zum Teil der Menschenwürde, oft aber auch zum Inhalt nationaler Autonomiebestrebungen. Sobald die gemeinsame Sprache nicht selbstverständliche Staats- und Freiheitsgrundlage ist, sucht die Rechtsordnung die Vielsprachigkeit durch Sprechregeln zu steuern; Sprache wird zum Gegenstand rechtlichen Regelns. Bestimmungen über Amts- und Arbeits- 
sprachen, über authentische Sprachen eines Rechtstextes und dessen Interpretation suchen durch Sprachkollisionsregeln ein Stück Spracheinheit zu ersetzen und Sprachminderheiten zu schützen. Das Recht, den eigenen Namen in der eigenen Muttersprache und Ortsnamen in der traditionellen Sprache fortführen zu dürfen, in den Schulen in der eigenen Muttersprache unterrichtet, in staatlichen Verwaltungs- und Gerichtsverfahren in der eigenen Sprache angesprochen zu werden und in derselben Sprache erwidern zu können, entwickelt sich zu einem der wichtigsten Minderheitenrechte.

Der kulturelle Zusammenklang von Sprache und Recht offenbart sich in der ersten Entstehensquelle für Recht, der guten Gewohnheit. Wenn die Gesetzestexte ursprünglich dadurch entstanden sind, dass das Gewohnheitsrecht aufgeschrieben, also vorgefundene Verhaltensregel in Sprache und Schriftform gefasst worden ist, wenn heute der Handelsbrauch, Ortsüblichkeit, die Grundsätze des ordentlichen Kaufmanns, die guten Sitten, Treu und Glauben, die öffentliche Ordnung, Observanz Entstehens- und Erkenntnisgrund für geltendes Recht sind, so begegnen sich Recht und Sprache in der gemeinsamen Wurzel einer in das Bewusstheit des Sprachlichen gehobenen Kulturgemeinschaft, die ein Verständigen und Verstehen möglich macht und damit die Bereitschaft zu allein sprachlicher Konfliktschlichtung als gemeinschaftskonstituierendes Prinzip anerkennt.

\section{Anschaulichkeit und Merkfähigkeit des Rechts}

Recht will allgemein wirken und muss deswegen allgemeinverständlich formuliert sein. Gesetzgebung sollte mit dem Denken eines Staatsphilosophen beginnen und mit der Sprache eines Rundfunksprechers enden. Die einfache, klare Sprache und die allgemeinverständliche Systematik ist Geltungsbedingung eines Gesetzes. Wenn das Gesetz die Sprache ihrer Adressaten spricht, stützt es sich auf deren Fähigkeit zum Begreifen, Ausdrücken, Verstehen.

Noch im 15. Jahrhundert lehnte Sebastian Brant eine besondere Anerkennung und ein Honorar für sein Werk „Daß Narrenschyff“ ab, weil er seine Sprachfähigkeit der Kulturgemeinschaft verdanke. Wer die deutsche Sprache mitgestaltet, übt ein Mitwirkungsrecht in dieser Sprachgemeinschaft aus, die auf Dialog und Gegenseitigkeit angelegt ist, die sich wechselseitig fördert und anspornt, die stets auf den Partner angewiesen bleibt, ohne den Gespräch und Sprache nicht möglich ist. Jacob Grimm hat in seiner Schrift von der Poesie im Recht (1816) dargelegt, dass Gesetz und Epos in Herkommen und Gewohnheit einen gemeinsamen Ursprung haben. 
Keinem Dichter gehört das Lied; wer singt, weiß es bloß fertiger und treuer zu singen; kein Richter darf neues Recht finden, er verwaltet Amt und Dienst der Rechte. Alles Recht könne, gleich der Sage, noch in seiner „Einfachheit und Poesieähnlichkeit" erkannt werden. Hier klingt ein Auftrag, eine Erwartung, eine Hoffnung an, das Recht möge im Bemühen um Sachlichkeit, Verlässlichkeit, Nachvollziehbarkeit nicht die Allgemeinverständlichkeit verlieren, nicht die werbende Kraft für Rechtsideale einbüßen.

Die Rechtsgeschichte ist voll von Formen, Gesten und Gebärden, die das Gesprochene unterstützen. Der Eid, der Treuebund, auch das sich Hinwerfen vor dem Herrscher macht eine Quelle des Rechts bewusst, die jenseits des Sprachlichen liegt. Mit dem Reichen der Hand zeigt der Gastgeber, dass er gegenüber dem Gast nichts - keine Waffe und keinen Vorbehalt - in der Hand hat. Diese Geste kann vielfach - in Herzlichkeit, Alltäglichkeit, Reserviertheit - abgestuft werden, sollte deshalb in der Französischen Revolution von der Einheitsgeste des Bruderkusses abgelöst werden. Das Hinwerfen des Handschuhs bekundet die Fehde, der Pantoffel ist Ausdruck der weiblichen Herrschaft. Recht stützt sich stets auf ein Erinnern, nicht nur auf Erfahrung. Jacob Grimm berichtet, dass Vertragspartner früher bei wichtigen Verträgen kleine Kinder - Menschen mit der noch größten Lebenserwartung - mitgenommen und sie unmittelbar nach Vertragsschluss grundlos geohrfeigt haben, damit diese Kinder sich verlässlich an das Geschehen des Vertragsschlusses erinnern mögen. Diese Frühformen eines Notariats sind heute etwas kultivierter.

Rechtssprichwörter suchen die Anschaulichkeit, die Merkfähigkeit des Rechtssatzes zu verbessern. Der Satz „Wo kein Hahn ist, kräht die Henne“ bringt die weibliche Erbfolge bei Fehlen eines männlichen Erben zum Ausdruck. "Wo sich der Esel wälzt, da muss er Haare lassen“ bezeichnet den Gerichtsstand des Delikts. Der Bürgermeister ist solange im Amt, als „der Meier in einem Sessel ungehalten sitzen kann".

Das Recht wurde einprägsam und einsichtig in Stabreimen (Haus und Hof, Land und Leute, Nacht und Nebel), Endreimen (Rat und Tat, schalten und walten, vereint und versteint), Wiederholungen (Acht und Bann, Kraft und Macht, kund und zu wissen) und durch die positive Aussage mit nachfolgender Negation (die Wahrheit sagen und die Lüge lassen). Heute hingegen umgibt sich die Rechtsprache mit einem Hauch kühler Geschäftsmäßigkeit, will nicht eine Sprache in aller Munde, kein mundgerechtes Recht sein, sondern die Rechtsaussage verlässlich jedem Adressaten gleich überbringen, damit dem Gleichheitssatz dienen.

Diese Rationalität der Rechtssprache soll die verbindliche Regel zumindest dem Juristen verlässlich überbringen, mit großer Wahrscheinlichkeit 
für alle Betroffenen dieselbe Rechtsfolge begründen. Sie soll die Rechtspflege von subjektiven Empfindungen, Neigungen und Vorurteilen des Rechtsinterpreten lösen; die Kontinuität des Rechts in der Stetigkeit der Rechtsmaßstäbe und ihrer Anwendung sichern; eine gesetzlich gelenkte Wirklichkeitssicht vermitteln, die im Blickwinkel und nach Vorgaben des Gesetzes die Realität erfasst und ermittelt. Sie baut auf klare Kompetenzverteilung zwischen vorsprechendem Gesetzgeber und nachsprechenden Interpreten, insbesondere der Verwaltung und der Rechtsprechung.

In diesem Bemühen um Nüchternheit und Prägnanz unterscheiden sich die verschiedenen Rechtssprachen. Die Verfassungssprache wirkt eher werbend-programmatisch, die Verwaltungssprache eher nüchtern anordnend. Dogmatisch geformte Sprachen des Zivil oder des Strafrechts folgen den Linien der Fachterminologie, die Sprache junger Disziplinen wie das Umwelt- oder das Datenschutzrecht sucht noch ihren Weg zwischen Allgemein-, Rechts- und Sachsprache. Regeln für die vorgefundene Wirklichkeit - wie das Polizeirecht - sind offen für reale Entwicklungen, Regeln für Kunstfiguren des Rechts - wie das Steuerrecht - verharren im Raum des Sollens.

In allen diesen Teilrechtssprachen herrscht die Tendenz, die Rechtssprache juristisch zu verselbständigen und damit mehr und mehr von der Alltagssprache abzuheben. Dadurch verliert die Gesetzessprache die Kraft, Rechtsbewusstsein zu schaffen oder zu vertiefen. Sie überfordert den Bürger, der in dieser Sprache Erklärungen, etwa Steuererklärungen, abgeben soll. Sie verführt den Gesetzesanwender, auf Allgemeinverständlichkeit zu verzichten, drängt ihn aber zugleich in eine Rolle des Dolmetschers zwischen Allgemein- und Rechtssprache, der er nicht immer entsprechen kann.

Wenn der Steuerpflichtige das Einkommensteuergesetz liest, um den für sein Einkommen geltenden Steuersatz zu erfahren, trifft er nicht auf den biblischen Zehnten, der ihm anschaulich macht, was er dem Staat schuldet. Vielmehr sagt $\$ 32 \mathrm{a}$ EStG dass die tarifliche Einkommensteuer - für ein Einkommen von 50.000 Euro - beträgt: „(228,74 · z + 2397) · z + 1038“, wobei „z“ „ein Zehntausendstel des 13.469 Euro übersteigenden Teils des auf einen vollen Euro-Betrag abgerundeten zu versteuernden Einkommens" ist. Das Gesetz verweigert das Gespräch mit dem Steuerpflichtigen.

\section{Rechtsetzen und Rechtsprechen}

Ein Gesetz spricht in Schriftlichkeit, kann das Gesagte deshalb nicht durch einen Sprecherverdeutlichen, der in seiner Tonlage das eine hervorhebt und 
das andere in den Hintergrund drängt, in seiner Sprechweise Zufriedenheit oder Empörung bekundet, Distanz oder Nähe herstellt, Wohlwollen oder Befremden ausdrückt, Gelassenheit oder Aufgeregtheit vermittelt. Der Sprecher kann einstimmen oder umstimmen, zu- oder gegenstimmen. Er kann lächeln oder die Stirn runzeln, flüstern oder lärmen, vertrauen oder argwöhnen.

Alles das kann das Gesetz nicht. Es scheint mit der Verkündung sprachlos zu werden. Doch der Rechtsstaat verweigert nicht das Gespräch, sondern steht seinem Adressaten weiterhin zum Dialog zur Verfügung. Er bietet ihm in der Rechtsprechung einen eigenen Gesprächspartner, der mit ihm über die ihn betreffenden generellen Rechtssätze spricht, ihre Wirkungen im individuellen Fall benennt, ihre Folgen rechtfertigt. Das Gesetz spricht von der Zukunft, regelt den zukünftigen, heute noch unbekannten Fall. Die Vorschrift ist Vorausschrift, der Vorrang und Vorbehalt des Gesetzes meinen auch seine Vorherigkeit. Die Rechtsprechung hingegen handelt von dem konkreten abgeschlossenen Fall, spricht den individuell Betroffenen an.

Eine Vorschrift, die in die Zukunft vorgreift, ist mit ihrer Verkündung schon veraltet. Als das Grundgesetz 1949 die Freiheit des „Rundfunks“ garantierte, musste diese Gewährleistung bald auf das Fernsehen erstreckt werden. Die Garantie der „Pressefreiheit“ war ursprünglich auf den technischen Druckvorgang des Pressens eines Schriftstücks angelegt, wird heute mit der Änderung der Medientechnik als eine allgemeine Druckfreiheit gedeutet werden müssen. Mit diesem Anspruch, heute rechtsverbindlich die Frage von morgen zu regeln, verschärft sich die Überlegung von Wittgenstein, ob die Sprache Kleid oder Verkleidung des Gedankens sei. Auch die für das Dichterwort gestellte Verantwortungsfrage Heinrich Bölls steigert sich: „Kaum ausgesprochen oder hingeschrieben, verwandeln sie (die Worte) sich und laden dem, der sie aussprach oder schrieb, eine Verantwortung auf, deren volle Last er nur selten tragen kann". Die Gewaltenteilung zwischen Gesetz und Rechtsprechung trennt Verantwortlichkeiten für das Aussprechen von Recht. Keiner könnte die Last, den Einzelfall aus einer allgemeinen Regel richtig zu lösen, allein tragen. Deshalb geht das Gesetz voraus, die Rechtsprechung folgt nach.

Dieses Nachdenken entwickelt sich, das zeigt unsere Sprache sehr deutlich, als Sprechvorgang. Die Rechtsprechung beginnt mit Widerspruch und Einspruch. Der Bürger erhebt Klage, sucht ein Urteil durch Berufung zu verbessern. Die Rechtsprechung entscheidet über Anspruch und Freispruch, gewährt in mündlicher Verhandlung rechtliches Gehör, hört auf den Wortlaut des Gesetzes, erwägt auch eine entsprechende Anwendung, spricht im Namen des Volkes. Der Spruchkörper entscheidet durch 
Abstimmen, verkündet sein Urteil. „Rede“ bedeutet ursprünglich „Rechenschaft" und "gerichtlicher Parteivortrag". Redner ist anfangs der Wortführer vor Gericht. Recht und Rede, nomos und Name, lex und Wort haben einen gemeinsamen Ursprung. Rechtsprechen ist nicht nur ein Nachsprechen von Vorgeschriebenem, sondern ein Nachdenken des Vorgeschriebenen.

\section{Recht auf Rädern}

Das Gesetz altert und kann veraltet sein. Deswegen sucht auch der Gesetzgeber seine Sprache auf diese Entwicklungsbedürftigkeit des Rechts auszurichten. Kunstfiguren des Rechts, z.B. Wahlen, Gerichtsverfahren, Kompetenzordnungen, Steuern veranlassen Rechtsfragen, die vorrangig aus einem gleichbleibenden Gesetzestext zu lösen sind. Handelt der Rechtssatz hingegen von Ergebnissen des Erkennens, z.B. das Arzneimittelrecht oder das Recht der technischen Sicherheit, so findet der Rechtssatz in der wissenschaftlichen Erfahrung und im jeweiligen Entwicklungsstand von Wissenschaft und Technik seinen wesentlichen, fortschreitenden Inhalt. Ist der Rechtssatz auf willentliche Weiterbildung angelegt, z.B. bei der Pflicht zur Stabilisierung des gesamtwirtschaftlichen Gleichgewichts, bei Einräumung eines Ermessens oder bei der Offenheit für einen Vertrag, so empfängt der Rechtssatz seinen Kern aus dem Willen der Beteiligten. Fragmentarische Rechtssätze, z.B. öffentliche Belange, das Wohl der Allgemeinheit, der wichtige Grund, die Billigkeit, deuten die gemeinte Verbindlichkeit lediglich an, fordern also eine Normenkonkretisierung von besonderer Intensität. Deswegen kann der Polizeibeamte die polizeiliche Generalklausel der öffentlichen Sicherheit und Ordnung so handhaben, dass das Singen des Kindes auf der Straße als Freiheitsausübung, dieselbe Tätigkeit im Vortragssaal als Störung qualifiziert wird; die leichte Kleidung am Strand als angemessen, beim Trauerakt als ordnungswidrig bewertet wird; die körperliche Attacke auf dem Rugbyplatz geduldet, beim anschließenden Festbankett unterbunden werden muss.

Auch der entwicklungsoffene Gesetzestext begegnet aber Anfragen an die Rechtsordnung, die er in seiner Aussage und seinem Sinn auch nicht annähernd voraussehen konnte. Dieses zeigt insbesondere das Glück der Wiedervereinigung Deutschlands, das zur Herstellung der inneren Einheit Deutschlands eine Handhabung des Grundgesetzes erforderlich machte, die 1949 so nicht bedacht werden konnte und in der sprachvermittelten Rechtskultur auch nicht vorausgesehen worden ist. Ist etwa ein Ehepaar in der ehemaligen DDR gegen seinen Willen geschieden worden, der Ehe- 
mann dann nach Ausweisung in den Westen eine neue Ehe eingegangen und Vater dreier Kinder geworden und beantragt die erste Ehefrau nunmehr die Feststellung der Nichtigkeit der Zwangsscheidung, so tut sich das Gericht mit der Feststellung der Rechtswidrigkeit der Zwangsscheidung leicht, mit der Feststellung der Nichtigkeitsfolge hingegen schwer, weil diese wohl bedeutet, dass der Ehemann in Bigamie lebt und die Ehelichkeit seiner Kinder gefährdet ist. Wenn ein Spion der DDR bei der Spionage in Westdeutschland erfolgreich war, deshalb von seinem Heimatstaat Honorar und Ehrung erwartet, nun aber feststellen muss, dass ausspionierender und ausspionierter Staat identisch geworden sind, so ist jedenfalls der Strafanspruch des wiedervereinigten Deutschlands gegen diesen Spion nicht mit herkömmlicher Härte durchzusetzen. Wenn der Mauerschütze auf den wehrlosen Flüchtling in der Spree schießt, weil er dazu „vergattert" worden ist, die auch für die DDR verbindliche Menschenrechtscharta hingegen einen solchen Schuss verbietet, macht sich die freiheitliche Demokratie der Gegenwart bewusst, dass die Normenlage in einer Diktatur das Berufen auf geltendes Recht oft untersagte, wenn es von der Staatsräson und von dem konkreten politischen Befehl überlagert worden ist. Wenn das Wahlrecht eine 5\%-Klausel kennt, die Anwendung dieser 5\%-Klausel für die erste gesamtdeutsche Wahl aber für die Parteien der ehemaligen DDR zur Folge hätte, dass sie die 5\% nur in ihrem angestammten Wählergebiet erreichen konnten, in Westdeutschland hingegen nicht einmal Kandidaten aufstellen, geschweige denn um Wähler werben konnten, so wirkt die 5\%-Klausel für diese Parteien als 23,7\%-Klausel und ist in dieser tatsächlichen Entwicklung verfassungswidrig. Diese wie auch weitere Fälle der Einigungsvertrag, die Bodenreform, die Warteschleife des öffentlichen Dienstes - mussten entschieden werden, ohne dass der Gesetzgeber die Entscheidung vorausgesehen hätte. Recht ist stets in Bewegung, stets auf Rädern.

Der Richter hat deswegen nicht die Aufgabe, gleichsam als Anwendungsautomat nur das zu wiederholen, was im Gesetz längst gesagt ist. Er beantwortet vielmehr eine Rechtsfrage am Maßstab des Gesetzes, die in ihrer Gegenwärtigkeit, Individualität und Besonderheit im Gesetz keine vollständige Antwort findet. Rechtsprechen empfängt im Gesetz seinen verbindlichen Maßstab, muss diesen aber vervollständigend und ergänzend auf den zu entscheidenden Fall anwenden. Vielfach ergeben sich dabei neue - gesetzlich angeleitete, aber das Gesetz weiterführende - Rechtsprinzipien, die von der höchstrichterlichen Rechtsprechung in Leitsätzen - in der sprachlichen Form eines Gesetzes - veröffentlicht und von der Rechtsgemeinschaft als verbindlich behandelt werden. 


\section{Rechtsänderung durch willentliche Umdeutung}

Das Gesetz überbringt Rechtsverbindlichkeiten, gerät in seinen Aussagen damit in den Einflussbereich von Sprachumdeutern und Sprachfälschern, die dem Rechtssatz einen für sie günstigen Inhalt geben wollen.

Während der Interpret eines Literaturtextes bei Fehlinterpretation seinen Gegenstand verfehlt, kann der Richter eines Gesetzestextes durch Fehlinterpretation seinen Gegenstand verändern. Der Interessent schlägt einen ihm günstigen Gebrauch der Rechtssprache vor; er neigt zu berechnender, gelegentlich zu verfälschender Verwendung der Rechtssprache, um den Inhalt des Gesetzes, zu dessen Änderung ihm die notwendige Mehrheit fehlt, durch Uminterpretation zu verändern. Er betreibt planmäßig eine bestimmte Sprechweise, um einer rechtlichen Aussage trotz gleichbleibenden Textes einen neuen Inhalt zu geben.

Der Rechtsordnung ist die Aufgabe gestellt, mit dem Sprachbetrug den Rechtsbetrug abzuwehren, die Änderbarkeit des Gesetzes allein der parlamentarischen Änderung der Gesetzesurkunde vorzubehalten. Gegenwärtig scheint die Rechtswirklichkeit für eine geplante Verwirrung von Rechtsinhalten trotz gleichbleibenden Gesetzestextes zugänglich: Der öffentliche Dienst soll durch Verwendung werbewirksamer Begriffe wie Partizipation oder Mitbestimmung von einer der Allgemeinheit dienenden Organisation zu einem Instrument der Bediensteten umgewidmet werden. Das Sozialrecht sucht die unserer Demokratie fremde Einheitsliste als „Friedenswahl“ zu beschönigen, um von dem Tatbestand einer Wahl ohne Alternative abzulenken. Eine Müllhalde wird als „Entsorgungspark“ präsentiert, die Bevorzugung der Kapitalerträge vor den Arbeitserträgen schlicht als Tarif,,spreizung“vermittelt, um die Bedeutung der Rechtsfrage zu vermindern. Das organisierte Willkürprinzip bei der Studienzulassung nennen wir Los,,verfahren“. Wenn wir statt von Todesschuss vom finalen Rettungsschuss sprechen, statt von Lohnerhöhung von Lohnangleichung reden, den Konjunktureinbruch als Null oder Minuswachstum beschönigen, müssen wir uns nicht nur fehlende Sprachdisziplin vorwerfen, sondern uns als Sprachfälscher rügen lassen. Wortfälschung ist eine Revolutionstaktik, die jede Rechtsordnung an ihrer empfindlichsten Stelle trifft.

Die Gegenwehr gegen eine Gesetzesverfremdung durch Sprachbetrug liegt nicht im Verbot verfälschender Sprechweisen. Eine sprachlich ungebundene Auseinandersetzung mit dem Recht ist für ein freies und entwicklungsfähiges Gemeinwesen unerlässlich. Die Grundidee eines freiheitlichen Rechtsstaates verbietet, für den allgemeinen Sprachgebrauch einen staatlich überwachten Firmen-, Marken- oder sonstigen Zeichenschutz einzuführen. 
Eine staatliche Kontrolle gegen unlauteren Wettbewerb bei der Interpretation von Rechtstexten ist dem Verfassungsrecht unbekannt und unerwünscht. Gerade das Gerichtsverfahren baut auf ein Gegeneinander von Kläger und Beklagtem, von Staatsanwalt und Rechtsanwalt, von Behörde und Hoheitsunterworfenem. Der Prozessbeteiligte ist bei seinem Rechtsvortrag - im Gegensatz zum Sachvortrag - nicht an Wahrheitspflichten gebunden. Die einzige Folge eines törichten und eines unredlichen Interpretationsvorschlages ist der Subsumtionsfehlschlag, also die Zurückweisung des Interpretationsvorschlags durch den verbindlich sprechenden Interpreten.

\section{Verantwortung für die Rechtssprache}

Welche Sprache spricht das Recht? Es wählt die Sprache der Rechtsgemeinschaft, für die sie Rechtsverbindlichkeiten begründen will, sucht möglichst unmissverständlich und nicht missdeutbar zu sprechen, vermittelt Rechtsgedanken in einer eigenen und doch verallgemeinerungsfähigen Sprache, lebt in Sprachlichkeit und Sprechbarkeit. Die Bestimmtheit der Rechtssprache findet ihre Grenze in dem Auftrag des Gesetzes, in die Zukunft vorzugreifen, also den noch unbekannten Fall mitzuregeln.

Die Allgemeinheit des Gesetzes trifft auf die Besonderheit des Einzelfalls, muss insoweit in der Gesetzesanwendung vervollständigt und ergänzt werden. Die moderne Sprache des Rechts bemüht sich vor allem um Nüchternheit, Sachlichkeit, Verlässlichkeit, vernachlässigt insoweit das anschauliche, dem demokratischen Staatsvolk entgegenkommende Sprechen. Seine Abstraktheit und Sachlichkeit schafft Distanz. Die Fülle, teilweise das Übermaß der Gesetze drohen inflationär den Wert des Gutes „Recht“ zu gefährden. Der Gesetzgeber sollte nur soviel sprechen, wie der Adressat des Gesetzes in seiner Gesprächsbereitschaft und Aufnahmefähigkeit entgegennehmen und verstehen kann.

Die Sprache des Rechts baut auf die Arbeitsteilung der Rechtsorgane. Das Gesetz spricht in einer allgemeinen Regel in die Zukunft. Die Verwaltung gibt die Antworten auf die Gegenwartsanfragen der Rechtsgemeinschaft. Die Rechtsprechung beurteilt den vergangenen Fall. So bleibt der Rechtsstaat ständig sprechfähig - in einer langfristig verbindlichen Regel, einem den Einzelfall regelnden Sprechakt, einem die Einheit und Fortbildung des Rechts gewährleistenden Richterspruch. Der Rechtsstaat wirkt mit der Gewalt der Sprache. Sie ist Ausdruck seiner Gestaltungsmacht, seines Wollen, seines Vermögens, seiner Selbstbindung. 


\section{Literatur}

Sebastian Brant, Daß Narrenschyff, 1494, abgedruckt in: Manfred Lemmer (Hrsg.), Das Narrenschiff, 2. Aufl., 1968.

BVerfGE 12, 205 (226) - 1. Rundfunkentscheidung.

BVerfGE 89, 155 (185) - Maastricht.

Iso Camartin, Nichts als Worte? Ein Plädoyer für Kleinsprachen, 1985.

Günter Dürig, Der deutsche Staat im Jahre 1945 und seither, VVDStRL, 13, 1954.

Jacob Grimm, Von der Poesie im Recht, in: Zeitschrift für geschichtliche Rechtswissenschaft, Band II, H. 1, 1816.

Bernhard Großfeld, Sprache und Recht, in: JZ 1984.

Peter Häberle, Sprachen-Artikel und Sprachprobleme in westlichen Verfassungsstaaten eine vergleichende Textstufenanalyse, 1990.

Hans Hattenhauer, Zur Geschichte der deutschen Rechts- und Gesetzessprache, in: Berichte aus den Sitzungen der Joachim Jungius-Gesellschaft der Wissenschaften e.V., Hamburg (1987).

Josef Isensee, Staat im Wort, 1995.

Rudolph von Jhering, Der Zweck im Recht, 2. Band, 4. Auflage, 1905.

Paul Kirchhof, Rechtsänderung durch geplanten Sprachgebrauch?, in: Dieter Wilke/ Harald Weber (Hrsg.), Gedächtnisschrift für Friedrich Klein, 1977.

Paul Kirchhof, Deutsche Sprache, in: Josef Isensee/Paul Kirchhof (Hrsg.), Handbuch des Staatsrechts der Bundesrepublik Deutschland, \$20, Band II, 3. Aufl., 2004.

Friedrich Kluge, Etymologisches Wörterbuch der deutschen Sprache, 24. Aufl., 2002.

Walther Merk, Werdegang und Wandlungen der deutschen Rechtssprache, 1933.

Mona Ozouf, Brüderlichkeit, in: François Furret/dies. (Hrsg.), Kritisches Wörterbuch der Französischen Revolution, Band 2, 1996.

Walter Otto, Erwartungen an die Rechts- und Verwaltungssprache der Zukunft, in: Muttersprache 91, 1982.

Leonhard Winkler, Deutsches Recht im Spiegel deutscher Sprichwörter, 1927.

Ludwig Wittgenstein, Tractatus logico-philosophicus, 1921, 4.002, abgedruckt in: ders., Schriften 1, 1969. 